6. Montori VM, Kleinbart J, Newman TB, Keitz S, Wyer PC, Moyer V, et al. Tips for learners of evidence-based medicine: 2. Measures of precision (confidence intervals). CMA7 2004;171 (6):611-5.

DOI:10.1503/cmaj.1050077

\section{Guidelines for STEMI}

W e commend Peter Bogaty and colleagues $^{1}$ for their Canadian adaptation of the ST-elevation myocardial infarction (STEMI) guidelines. They have appropriately emphasized the importance of time to reperfusion, whether thrombolysis or primary percutaneous coronary intervention (PCI) is used. Although primary PCI may be superior to thrombolysis when performed in a timely manner, this benefit may be attenuated or lost altogether when PCI is delayed more than 60 minutes. ${ }^{2}$ However, it may be possible to derive the benefits of primary PCI without the inherent treatment delay by administering thrombolysis followed by immediate transfer for PCI. This strategy, termed "facilitated PCI," may be the optimal mode of reperfusion for many patients in Canada, where interventional centres are regionalized. Although early studies failed to show a benefit of routine PCI immediately after thrombolysis, ${ }^{3}$ PCI technology has changed considerably in recent years. More recent studies have indicated that facilitated PCI may indeed be safe and effective, ${ }^{4}$ but larger studies are needed to provide definitive answers.

The TRANSFER-AMI trial, initiated by Canadian investigators and funded by the Canadian Institutes of Health Research, will randomly assign approximately 1200 high-risk STEMI patients treated with thrombolysis in non-PCI hospitals to be transferred immediately for facilitated PCI or to receive standard care. This study could have a significant impact on the treatment of STEMI in Canada, and we strongly encourage Canadian centres to participate (for further information, see the Web site of the Canadian Heart Research Centre, www.chrc.net).
Warren J. Cantor

St. Michael's Hospital

Toronto, Ont.

Laurie J. Morrison

Sunnybrook and Women's College

Health Sciences Centre

Toronto, Ont.

\section{References}

1. Bogaty P, Buller CE, Dorian P, O'Neill BJ, Armstrong PW. Applying the new STEMI guidelines: 1. Reperfusion in acute ST-segment elevation myocardial infarction. CMA7 2004;171 (9):1039-41.

2. Nallamothu BK, Bates ER. Percutaneous coronary intervention versus fibrinolytic therapy in acute myocardial infarction: Is timing (almost) everything? Am 7 Cardiol 2003;92:824-6.

3. Michels KB, Yusuf S. Does PTCA in acute myocardial infarction affect mortality and reinfarction rates? A quantitative overview (metaanalysis) of the randomized clinical trials. Circulation 1995;91:476-85.

4. Le May MR, Labinaz M, Turek M, Leddy D, McKibbin T, Quinn B, et al. Combined Angioplasty and Pharmacological Intervention versus Thrombolytics Alone in Acute Myocardial Infarction (CAPITAL AMI) Study: six-month results [abstract]. Can 7 Cardiol 2004;20(Suppl D):151-D

Competing interests: None declared for Dr. Morrison. Dr. Cantor has received research funding and honoraria from Hoffmann-La Roche.

DOI:10.1503/cmaj.1041728

$\mathrm{P}$ eter Bogaty and colleagues, ${ }^{1}$ in their review of the American College of Cardiology/American Heart Association STEMI guidelines from a Canadian perspective, recommend transfer of STEMI patients with Killip class $3 / 4$ or other high-risk features of acute myocardial infarction for PCI, if such intervention is reliably available within 60 minutes. However, achieving a 60-minute transfer imposes significant challenges for emergency medical services (EMS) that the authors have not considered. Several studies examining interfacility transfer for primary PCI, operating under rigorous study protocols, were able to achieve randomization-to-balloon times of 80 to
122 minutes, ${ }^{2-5}$ which suggests that meeting a 60-minute target may be difficult in everyday practice.

The following recommendations would help to safely achieve this target:

- The paramedics caring for the patient should be capable of advanced life support (ALS) interventions, as some of the patients may experience the complications of STEMI while in transit. ${ }^{4}$ Therefore, EMS dispatch should provide an ALS-crewed vehicle in the same time frame as would apply for a critical 9-1-1 call (in our system, this would be 8 minutes, 59 seconds). Alternatively, the same ambulance that brought the patient to the emergency department, if its crew is capable of providing ALS, should be used to transfer the patient.

- A PCI "hot link" should exist between the referring and receiving institutions. The PCI centre should accept referrals without question and should reassess for PCI suitability on arrival.

- Patients should be taken directly to the catheterization suite, without a stop in the receiving emergency department.

We feel that a 60-minute target for transfer is unlikely to be met without specific optimization of EMS and hospital systems. The absence of such optimization will inevitably lead to failure and abandonment of a strategy that has the potential to lessen morbidity and mortality.

\section{Cathal O'Donnell \\ Richard Verbeek}

Base Hospital Programme

Sunnybrook and Women's College

Health Sciences Centre

Toronto, Ont. 